\title{
Strates
}

STRATES Matériaux pour la recherche en sciences sociales

Hors-série | 2002

Parcours dans la recherche urbaine, Michel Rochefort, un géographe engagé

\section{Justice et territoire une réflexion à partir du cas brésilien}

\section{Bernard Bret}

\section{(2)enEdition}

\section{Journals}

Édition électronique

URL : http://journals.openedition.org/strates/531

DOI : $10.4000 /$ strates.531

ISSN : $1777-5442$

Éditeur

Laboratoire Ladyss

Édition imprimée

Date de publication : 1 janvier 2002

ISSN : 0768-8067

Référence électronique

Bernard Bret, « Justice et territoire une réflexion à partir du cas brésilien », Strates [En ligne], Hors-série | 2002, mis en ligne le 02 mai 2005, consulté le 08 septembre 2020. URL : http:// journals.openedition.org/strates/531 ; DOI : https://doi.org/10.4000/strates.531

Ce document a été généré automatiquement le 8 septembre 2020.

Tous droits réservés 


\title{
Justice et territoire une réflexion à partir du cas brésilien
}

\author{
Bernard Bret
}

1 S'il est un thème classique de la géographie du Brésil qui mérite d'être revisité, c'est bien celui des inégalités régionales. Il est classique parce que, pour une discipline qui étudie la différenciation spatiale, le territoire brésilien offre un terrain d'observation d'une netteté peu commune. Dans ce pays émergent qui figure aujourd'hui parmi les grands exportateurs de produits industriels et qui compte avec São Paulo une métropole mondiale, l'extrême pauvreté touche partout une proportion importante de la population et marque durablement la région Nordeste. Il est à revisiter parce qu'il pose d'une façon éclairante le problème des relations qu'entretiennent les sciences humaines avec certaines notions qui ne ressortissent pas toujours au champ scientifique et qui, pour certaines, relèvent plutôt de l'éthique.

2 Qu'il s'agisse d'économistes et de sociologues attentifs aux mécanismes des inégalités sociales, ou de géographes, plus sensibles aux inégalités spatiales, cherchant à comprendre comment les disparités socio-économiques s'expriment dans l'organisation du territoire et se nourrissent de cette organisation, une référence implicite est souvent présente dans leurs travaux à la notion de justice. On étudie l'inégalité en tenant comme allant de soi que l'égalité vaut mieux sur le plan social que l'inégalité. On étudie le développement en le tenant pour préférable au sousdéveloppement sans qu'il soit jugé nécessaire de démontrer une pareille évidence. Bien entendu, une telle attitude est respectable et correspond à la sympathie que de nombreux chercheurs éprouvent à l'égard des victimes de cette inégalité. Mais le problème qui demeure entier est celui de la validité scientifique d'une telle posture. La préoccupation pour la justice est-elle un atout pour comprendre le fait social, ou peutelle devenir un obstacle pour l'analyse ? Vaut-il mieux se départir de toute tentation de juger et observer les acteurs sociaux avec l'impartialité de l'entomologiste examinant les comportements des populations animales?

On comprend bien, certes, que deux domaines distincts existent. L'un relève du savoir et requiert une certaine distance entre l'observateur et l'objet d'étude. L'autre relève de 
l'action et implique l'engagement au nom de certaines valeurs. Mais on pressent aussi que les choses ne sont pas si simples et que le dédoublement du même individu entre celui qui cherche et celui qui agit conduit à des contorsions difficiles. Il existe en fait une autre manière de poser le problème. Pour la comprendre et voir qu'elle est plus productive, un rappel de terminologie peut aider, tant il est vrai que la façon de nommer les choses n'est jamais étrangère à l'idée que l'on s'en fait. C'est la situation latino-américaine qui a fait naître le mot de maldéveloppement : pourquoi introduire ce préfixe mal, sinon pour introduire explicitement une notion de valeur et dire dans le mot lui-même que ce développement est mauvais, un peu comme les médecins parlent de malformation pour décrire un organisme non conforme au modèle biologique. Mais la médecine n'est pas seulement un savoir, c'est aussi une technique qui cherche à guérir; pour elle, parler de malformation est donc logiquement comparer un cas clinique à une norme fixée par la nature, et établir que la santé est préférable à la maladie entre logiquement dans sa démarche qui est à la fois de connaissance et d'intervention. Est-on fondé à faire de même dans les sciences humaines? Est-ce ici légitime de parler de maldéveloppement, c'est-à-dire d'analyser une situation socioéconomique en portant sur elle un jugement en référence à des valeurs éthiques?

4 Cette interrogation que tout chercheur en sciences sociales s'est un jour posée - du moins peut-on l'espérer - trouve un élément de réponse dans le fait que la notion de justice est un thème de la philosophie politique et constitue donc un critère recevable de compréhension des logiques sociales. Il ne s'agit pas de prendre parti pour les uns contre d'autres, de se départir du devoir de neutralité qui est le gage de la validité scientifique du discours. Mais il est possible de penser l'éthique et de retenir la notion de justice pour éclairer les faits sociaux et en appréhender la logique.

Le territoire est constitutif du lien socialLa justice : une notion aux champs d'application multiples

5 Si la notion de justice peut guider le raisonnement géographique, c'est parce que le territoire est lui-même considéré comme un élément du lien social. Étudier les rapports entre les hommes passe donc aussi par l'étude de leurs rapports respectifs à un espace partagé, disputé pour sa valeur économique et doté d'une charge symbolique.

6 Parler de justice requiert de préciser les divers champs de validité de la notion. Le mot s'entend le plus immédiatement comme justice sociale. Il s'applique ainsi aux relations entre les hommes et désigne une certaine égalité (certaine, car l'égalité totale, à supposer qu'elle soit possible, n'est pas nécessairement une condition sine qua non d'un ordre social juste) dans leurs conditions de vie. La distribution de la richesse, patrimoine et revenu, en est le critère principal, mais aussi l'accès aux prestations touchant la santé, l'éducation, la culture.

7 Cette affirmation se décline de multiples façons, s'enrichissant à chaque fois d'un contenu nouveau. Précision qui n'a pas encore fait totalement son chemin et dont on s'étonnera sans doute dans les siècles futurs qu'une telle évidence ait eu autant de mal à s'imposer : l'égalité entre les hommes et les femmes est une composante essentielle de la justice dans une humanité qui est masculine et féminine. On parle bien ici d'égalité pour distinguer cette donnée de toutes les autres et signifier que l'appartenance à un sexe plutôt qu'à l'autre ne doit pas être un facteur aggravant les inégalités qui peuvent exister entre les personnes pour d'autres raisons. Qu'il ne soit pas toujours facile d'isoler ce qui relève de la division sexuelle dans les situations réelles n'invalide pas la méthode, mais au contraire souligne la nécessité de disposer en la matière de principes 
simples et nets susceptibles d'éclairer des situations complexes. Toutefois, on doit s'interroger sur la dimension géographique de l'inégalité sociale ou entre les sexes. De tels faits, du moins certains d'entre eux, peuvent être mesurés et localisés. Est-ce à dire que leur approche relève d'une démarche géographique ? Cela demeure discutable tant qu'il s'agit seulement de cartographier les indicateurs statistiques: savoir où se produisent les faits, dans quel sens varient les gradients et où passent les lignes de fracture est bien entendu utile, mais c'est davantage une étape préalable à l'analyse géographique que l'analyse elle-même si le territoire est vu comme le support physique des faits sociaux décrits sans être incorporé dans un raisonnement explicatif.

Le troisième champ d'application de la notion introduit la dimension du temps. On peut parler alors de justice intergénérationnelle. Chacun n'a qu'une vie et se sent donc concerné d'abord par ce qui se passe dans son époque et comporte des conséquences pour lui et ses descendants immédiats. Cette restriction de la réflexion à un temps court à l'échelle de l'humanité a longtemps occulté les injustices qui traversent les générations et qui tiennent aux décalages historiques entre les investissements, au sens le plus large du mot, et leur rentabilisation. Chaque génération hérite de ce qu'ont fait, en positif ou en négatif, les générations antérieures, et laisse un patrimoine naturel et culturel aux générations ultérieures. On verra plus loin comment ce très simple constat est productif pour penser l'accumulation primitive et la question environnementale.

La justice entre les lieux n'est pas moins importante. Peut-être plus familière à qui s'occupe de géographie, elle ne prend sens que si elle englobe le fait humain, et c'est le terme de justice socio-spatiale, dû à Alain Reynaud ${ }^{1}$, qui exprime le mieux le phénomène. Comme il a été fait plus haut pour la division sexuelle, il convient d'écarter provisoirement du raisonnement les éléments extérieurs au facteur considéré et d'isoler ce qui relève du territoire dans ce qui est porteur d'inégalité entre les hommes. L'idée de justice, et d'injustice, socio-spatiale exprime alors le fait que la localisation peut favoriser indûment tel ou tel groupe, et que les relations qu'entretiennent les lieux interfèrent avec les relations qu'entretiennent les hommes.

La différenciation spatiale

Il découle de ce qui précède que la différenciation spatiale, c'est-à-dire finalement ce dont s'occupe la géographie, offre un champ d'observation privilégié de la justice et de l'injustice. On laissera provisoirement en suspens la question de savoir si certaines inégalités sont admissibles et on notera que différenciation ne signifie pas inégalité... précaution de méthode qui met en place trois termes fondamentaux pour le sujet: différence, inégalité, injustice. Quand la différence devient-elle inégalité ? Quand l'inégalité devient-elle injustice?

11 Les configurations géographiques produites par les relations économiques et sociales sont connues. Le modèle centre-périphérie en offre une synthèse pertinente et constitue donc un outil efficace pour analyser les territoires. Les flux qui relient le centre, où s'accumulent les facteurs de production et les instances de pouvoir, et la périphérie, fournisseuse de biens, d'hommes, de capitaux, et destinataire de certaines retombées du centre, produisent une dissymétrie (sans cette dissymétrie, le modèle centre-périphérie traduirait simplement la différenciation spatiale produite par des raisons techniques, et non pas l'inégalité), éventuellement vectrice d'injustice entre les acteurs sociaux partenaires. L'important est de savoir si le mécanisme induit un transfert de valeur, à travers le prix des biens échangés, la rémunération du travail qui les a produits, les migrations aboutissant à la valorisation en certains lieux d'un capital 
humain formé ailleurs, la répartition de l'effort fiscal et de la dépense publique, la mobilité des capitaux selon l'inégale qualité de l'environnement économique et des perspectives de valorisation.

Justice et développement au BrésilRepenser le modèle centre et périphérie en s'inspirant de John Rawls

Brésil, pays de contrastes... Le territoire brésilien offre une image presque caricaturale du modèle centre-périphérie. Reste à saisir le ressort social de cette inégalité sociale et régionale répétée à l'envi au point de tourner au cliché.

Il s'agit d'abord de comprendre comment l'inégalité régionale est le résultat de deux dynamiques sociales opposées, produites l'une par l'injustice sociale maximum et l'autre par une moindre inégalité entre les hommes. L'Histoire invite dans le cas présent à commencer par la périphérie car le Nordeste fut la première région brésilienne à se construire, en l'occurrence autour de la production sucrière. C'est avec le cycle du sucre de canne (XVI ${ }^{e}$-XVII ${ }^{e}$ siècles) que les structures économiques, sociales et culturelles se sont mises en place. Plusieurs livres connus ont assez décrit la période pour qu'on s'autorise ici à ne pas en reprendre la narration et qu'on se limite au repérage de la tendance de longue durée qui a fait de cet espace une périphérie. L'interprétation ici avancée est que cette mise en périphérie a été facilitée par l'injustice sociale interne. Le fait décisif est la colonisation esclavagiste : l'exploitation totale de la main-d'œuvre a fait la fortune d'une petite minorité de planteurs et, audelà des mers, de négociants portugais et hollandais, et a fait sur place la ruine de la région. En effet, l'inégalité extrême de la distribution du revenu (les esclaves n'ont rien, les planteurs ont tout... moins ce que prélèvent les Européens) a empêché la formation d'un marché solvable et a donc interdit la diversification de l'appareil productif. En empêchant la fixation sur place des fruits du travail et en orientant l'investissement vers les seules exportations, le système esclavagiste a été la matrice du sousdéveloppement régional. Que l'injustice ait produit le sous-développement est finalement cohérent avec les mots employés : injustice ne va pas avec développement.

Le point important consiste donc dans le lien qui existe entre l'expropriation sociale des fruits du travail du fait du rapport de forces interne entre les classes sociales, et l'expropriation spatiale du surplus économique du fait du rapport de forces entre les sociétés globales.

Si, plus tard, la région de São Paulo a pu s'ériger comme centre, c'est grâce à une structure sociale tout à fait différente. La région s'est construite avec le cycle du café à partir du milieu du XIX ${ }^{\mathrm{e}}$ siècle. L'esclavage était encore en vigueur (l'abolition aura lieu seulement en 1888), de telle sorte que les plus anciennes plantations ont connu le travail servile. Ce système était condamné à terme, non seulement parce que l'idée de l'égalité des hommes faisait son chemin dans l'opinion publique, mais aussi parce que d'autres formes de mise au travail apparaissaient plus modernes et plus efficaces. Aussi, la région pauliste s'est construite principalement sur un système de travail libre. Ce fut la condition pour l'arrivée des migrants européens, italiens surtout, venus tenter leur chance dans une région exempte de l'héritage esclavagiste qui pesait si fort sur l'économie et la société du Nordeste, même une fois les esclaves libérés. Sans qu'il soit nécessaire de redire ici l'histoire de ce front pionnier du café, si brillamment analysé naguère par Pierre Monbeig ${ }^{2}$, il faut retenir qu'il a été l'occasion de former une classe moyenne, et que cette classe moyenne a rendu possible l'industrialisation lorsque la crise caféière des années 1930 fit de la reconversion économique un impératif. Cette 
reconversion économique régionale est passée par une reconversion du capital foncier en capital industriel. Encore fallait-il que la terre trouve des acheteurs : en se portant acquéreurs, fût-ce à crédit, les anciens colonos des fazendas montraient une relative aisance matérielle et apportaient confirmation que les conditions faites au travail dans le cycle du café pauliste n'avaient heureusement rien à voir avec celles qui avaient eu cours lors du cycle du sucre nordestin. La seconde condition de réussite de la reconversion économique était que les biens industriels trouvent preneurs, puisque les investisseurs misaient sur le marché intérieur, dans une stratégie analysée depuis lors comme une substitution d'importations: le marché solvable se trouvait précisément dans cette classe moyenne de producteurs agricoles qui avaient accédé depuis peu à la propriété foncière, grossie très vite par les salariés urbains dès lors qu'une dynamique de développement avait pu démarrer.

Un cercle vertueux de croissance était donc enclenché à São Paulo, explicable fondamentalement parce que les détenteurs de capitaux misaient sur la région.

Deux trajectoires économiques s'opposent donc nettement. L'une est produite par l'esclavagisme et ses séquelles et débouche sur le sous-développement. L'autre est produite par le travail libre et génère le développement. De ce contraste, on est tenté de conclure à l'efficacité de la justice, dans la mesure où une distribution plus égalitaire du revenu paraît être la condition pour fixer sur place les capacités d'investissement, et pour donner à une société les bases solides d'un marché solvable, et donc les opportunités d'investissement.

Cet élément de conclusion appelle pourtant débat. Dans aucune des deux situations régionales étudiées, et nulle part ailleurs dans le monde, qu'il soit développé ou sousdéveloppé, l'égalité sociale n'est atteinte. L'idée de justice continue à figurer parmi les utopies positives qui disent la marche à suivre et l'idéal vers quoi il faut tendre, mais le temps est encore lointain, s'il arrive un jour, où elle trouvera une pleine réalisation dans l'organisation de la vie sociale. Par conséquent, dire que la justice est efficace est une affirmation simpliste. Encore faudrait-il préciser ce que l'on entend par justice et dire pour qui et en référence à quoi l'on parle d'efficacité : la tautologie n'est en effet pas loin si l'on retient que le développement implique une croissance économique pour tous et si l'on conclut que la justice est efficace pour le développement... ce qui, à la limite, revient à dire que la justice est efficace pour la justice !

19 Mais cette affirmation n'est pas une impasse pour le raisonnement. De même que la justice parfaite n'existe nulle part, le développement total est chose inconnue et même difficile à concevoir. Cela n'empêche pas de constater qu'il existe des sociétés plus justes que d'autres, et des économies plus développées que d'autres. Il n'est donc pas vain de s'interroger sur les conséquences de plus ou moins de justice pour plus ou moins de développement, ce qui modifie la démarche initiale et conduit, puisque l'inégalité est partout, à formuler la question en des termes nouveaux: à quelles conditions l'inégalité est-elle efficace pour le mieux-être de tous?

Il ne manquerait pas de privilégiés pour s'engouffrer dans ce point de l'argumentation et tenter d'y trouver matière à légitimer leur position sociale. Combien de fois, au Brésil, a-t-on entendu les nantis dire qu'il fallait faire grossir le gâteau avant de le partager, signifiant par là que la distribution du revenu n'était pas à l'ordre du jour tant que l'économie nationale n'avait pas atteint un niveau supérieur à celui du moment. S'il est montré par les faits que la croissance écarte en général les revenus dans un premier temps et les rapproche dans un second temps, se satisfaire de l'inégalité, quelle qu'en 
soit l'intensité, au motif qu'elle serait une nécessité historique, relève plus de la compétition pour le partage de la croissance que d'une démarche intellectuelle, bien que les théories utilitaristes puissent venir à la rescousse pour habiller d'un discours à prétention rationnelle la volonté des riches de ne pas partager avec les pauvres.

21 John Rawls propose une réponse à cette difficile question. En considérant que l'inégalité n'est pas nécessairement contraire à la justice, pour autant qu'elle soit " attachée à des positions et à des fonctions ouvertes à tous et surtout qu'elle soit à l'avantage de chacun $\|^{3}$, il définit l'injustice comme « les inégalités qui ne bénéficient pas à tous $»^{4}$. Cette affirmation invite à se demander si le développement, qui est par définition un développement pour tous, est compatible avec l'inégalité des conditions et requiert au moins provisoirement une certaine inégalité des revenus. Si le Nordeste s'est enfoncé dans le sous-développement, c'est parce qu'il y avait trop d'inégalité dans sa structure sociale. Si la région de São Paulo, elle, s'est développée, est-ce malgré l'inégalité qui y existait aussi à un degré moindre ou parce que cette inégalité existait à un degré moindre, en l'occurrence mieux calé aux nécessités de l'accumulation et donc plus efficace pour le développement. On a vu qu'une extrême concentration des revenus décourageait l'investissement productif sur place. On peut se demander si, à l'inverse, l'égalitarisme ne réduirait pas la capacité d'épargne et n'empêcherait pas lui aussi la formation du capital productif, sauf à imaginer une société parfaite où l'égalité totale irait de pair avec l'acceptation consensuelle d'un prélèvement destiné à financer des investissements choisis démocratiquement... Le principe de réalité invite à la prudence puisque l'Histoire a montré la faillite des tentatives qui se réclamaient d'une telle société, même si l'utopie positive, elle, n'a pas nécessairement failli puisque aucune de ces expériences historiques ne comportait une véritable égalité sociale, ni surtout la démocratie.

Dans les situations réelles, et non pas dans celles que l'on se plait à imaginer, tout se passe comme si l'inégalité était constitutive de la dynamique sociale, elle-même constitutive du processus de développement. Or, bien que l'accumulation des richesses ne soit pas une condition de la justice (là encore, John Rawls a raison quand il écrit : "C'est une erreur de croire qu'une société juste et bonne devrait aller de pair avec un haut niveau de vie matériel. Ce dont les hommes ont besoin, c'est d'un travail ayant un sens, en association libre avec d'autres travailleurs, dans le cadre d'institutions de base justes. $»^{5}$ ), le développement doit être tenu pour un besoin dès lors qu'il est possible. Et on arrive à ce paradoxe que le développement est le fruit d'inégalités favorables, par leur nature et leur intensité modérée, à la diffusion des effets de la croissance dans l'ensemble du corps social. Le terme d'usage courant de développement inégal qui dit d'abord l'inégalité géographique, suggère donc aussi que le développement est nécessairement inégal dès lors qu'il est produit par une société elle-même inégalitaire.

Échelles et discontinuités : approche spatiale des effets de seuil

Plus ou moins favorisé par la structure sociale et l'intérêt que les groupes dominants ont ou n'ont pas à jouer la carte du développement régional et donc à investir ou à ne pas investir sur place, le processus d'accumulation se réalise ainsi sur des territoires qui, bornés par des limites politico-administratives, ne sont pas pour autant isolés les uns des autres, mais au contraire reliés par des flux. Ces flux constituent les vecteurs de la délocalisation des facteurs de production, capital et force de travail : leur dissymétrie produit le binôme centre-périphérie. 

structures différentes, l'une très inégalitaire et l'autre plus égalitaire, entrent en relation. La question consiste dans ceci : la justice apparente qui existe dans l'une des deux formations est-elle rendue possible par l'injustice qui sévit dans l'autre. À l'échelle mondiale, cette question renvoie au débat déjà ancien, mais toujours actuel car il n'a jamais reçu de réponse définitive, de l'échange inégal ${ }^{6}$. Par divers mécanismes, dont le niveau des prix n'est qu'un parmi d'autres et renvoie au niveau de rémunération du travail, y a-t-il transfert de valeur d'une formation socio-spatiale à une autre, permettant à cette dernière de réaliser une large distribution du revenu, l'apparente justice qui y règne à l'échelle locale ou régionale étant financée en réalité par l'injustice observable à une échelle internationale?

Comment les choses se passent-elles à l'intérieur d'un même territoire national lorsque, comme au Brésil, les structures sociales sont bien différentes d'une région à l'autre ? Celso Furtado a donné pour le Brésil une explication éclairante. Sous le nom d' effet triangulaire (les trois sommets du triangle étant le Nordeste, la région de São Paulo et l'étranger), il a montré comment la protection douanière dont le pays s'est entouré pour réussir son industrialisation a eu des effets opposés pour les différentes régions, précisément parce qu'elles avaient des structures sociales différentes. Le protectionnisme a profité à São Paulo et lui a permis de s'ériger en centre parce que la distribution du revenu y avait rendu possible l'investissement industriel. Le Nordeste, lui, fidèle à sa tradition agro-exportatrice et contraint de se fournir en biens manufacturés paulistes à des prix supérieurs à ceux du marché mondial, a contribué pour une part significative à l'essor du Sudeste. Il demeure vrai que le ressort principal qui a permis le décollage de São Paulo se trouve dans sa dynamique sociale propre. Il n'empêche que le transfert de richesse réalisé aux dépens du Nordeste a creusé l'écart, le mécanisme d'intérêt composé produisant à terme une accumulation forte dans le centre et $\mathrm{y}$ autorisant des retombées financières sur la population.

Le raisonnement gagne donc à être exprimé, non pas en termes de régions, ce qui pourrait être compris à tort comme de simples espaces, mais plus explicitement en termes de formations socio-spatiales ${ }^{7}$. Cela revient à croiser les phénomènes spatiaux et les phénomènes sociaux, sans quoi la géographie n'aurait pas d'existence en tant que science sociale. Il faut se garder de parler de régions riches et de régions pauvres, comme si les premières ne comptaient que des riches et les secondes que des pauvres! Certes, les moyennes ne sont pas sans signification, mais la réalité est que la richesse et la pauvreté se combinent en tout lieu et que s'exprimer en termes d'espace comporte toujours le risque de gommer dans le discours les inégalités sociales internes à chaque territoire. La remarque paraîtra peut-être superfétatoire : elle ne l'est pas si elle aide à décrypter les revendications régionales issues des groupes sociaux dominants, les seuls souvent à pouvoir se faire entendre, qui utilisent en fait le problème régional pour s'attirer les bontés des pouvoirs publics.

La question de la justice socio-spatiale appelle donc un raisonnement pluriscalaire. Si la périphérie nordestine est, en moyenne, pauvre, c'est parce qu'une minorité riche y assoit sa richesse sur la mise en périphérie de la région et se pose à l'interne comme un centre. Que le centre pauliste soit, en moyenne, riche, cela n'empêche pas qu'il comporte une périphérie interne modeste ou pauvre, dont le sort est adouci par les redistributions que permet l'exploitation de la périphérie externe, mais dont le nombre est agrandi par les migrations venues précisément de cette périphérie exploitée 
(phénomène parfois appelé nordestinisation du Sudeste). La richesse attire en effet la pauvreté : il faut se rapprocher du lieu de l'accumulation pour trouver un emploi, ou, à défaut, vendre des services non qualifiés aux riches. D'une façon ou d'une autre, le centre assure mieux que la périphérie les stratégies de survie des pauvres.

L'analyse géographique se fait donc très complexe dès lors qu'elle retient l'inégalité spatiale comme constitutive de l'inégalité sociale. Puisqu'on retient les flux comme moteurs dans le processus d'enrichissement inégal, il faut inscrire le raisonnement dans un espace qui n'est pas neutre, mais qui est un territoire maillé. Les limites administratives constituent des discontinuités dans les mécanismes de prélèvement et de prestation. C'est en quoi le maillage est un enjeu de pouvoir et non seulement une nécessité technique. C'est pourquoi aussi la géographie politique peut apporter une utile contribution à l'intelligence de la justice.

Aménagement et projet de société

La politique d'aménagement du territoire, indissociable d'un projet de société, est une politique au sens plein du mot: de la même façon que l'on porte appréciation sur une politique sociale en fonction de valeurs éthiques, on est fondé à juger des politiques territoriales et des mesures d'aménagement selon qu'elles sont porteuses ou non de plus de justice entre les hommes.

Principe de réparation ou modernisation de l'injustice?

Une fois le constat établi que la différenciation spatiale recouvre une inégalité régionale et que certains hommes, indépendamment de leur place dans la hiérarchie sociale et donc des injustices dont ils peuvent s'estimer être victimes par ailleurs, se trouvent défavorisés par rapport à d'autres du fait du lieu où ils vivent, on peut parler d'injustice socio-spatiale. Est souhaitable alors une action correctrice. L'État, en tant que garant des intérêts collectifs, peut agir directement par l'allocation des dépenses publiques et indirectement par des avantages particuliers, notamment fiscaux, accordés aux particuliers, entreprises et personnes physiques, engageant des actions jugées utiles dans les régions défavorisées. Ce dispositif donne parfois matière à une réglementation compliquée, mais il est simple dans son principe : il correspond à ce que John Rawls appelle principe de réparation ${ }^{8}$. C'est, pour reprendre un slogan expressif, donner plus à ceux qui ont moins. Toutefois, l'idée, chez John Rawls, ne débouche pas sur un égalitarisme qui gommerait toute inégalité, car seules doivent être combattues les inégalités qui sont contraire à la justice, c'est-à-dire qui sont infondées et/ou qui ne sont pas productrices d'un mieux-être pour tous.

31 Si l'on comprend bien en quoi une politique sociale peut s'adresser à ceux que le sort a atteints (les handicapés ou les malades), à ceux que l'évolution économique pénalise (les demandeurs d'emploi) ou encore à ceux qui supportent des charges utiles à tous sur le long terme (les chargés de famille), la mise en œuvre du principe de réparation est plus délicate à interpréter quand il s'agit du territoire. Certes, les politiques d'aménagement se réclament du principe de la justice pour motiver tout avantage particulier accordé à une zone. Mais il y aurait quelque naïveté à se référer aux seules déclarations d'intention en faveur de la justice et qui font partie du jeu politique : a-ton jamais vu un gouvernement dire autre chose et vendre à l'opinion publique un programme qui serait explicitement présenté comme devant produire plus d'injustice?

32 Si la notion de justice est un critère recevable pour analyser les politiques d'aménagement du territoire, c'est donc bien parce que ces politiques fondent leur 
légitimité sur la justice elle-même et ne peuvent se dérober à l'appréciation des résultats obtenus au regard des objectifs annoncés.

Le cas brésilien offre en la matière un vaste champ de réflexion. On sait que l'action publique au profit du Nordeste s'est accélérée en 1960, lorsque fut créée la Sudene Surintendance du développement du Nordeste - sur les idées de l'économiste Celso Furtado. Le contexte de la Cepal - Commission économique pour l'Amérique latine - et l'expérience, en Italie, de la Cassa per il Mezzogiorno, plaidaient pour que l'État intervienne comme instance de régulation territoriale, ce qui fut fait aussi en d'autres pays (en France, la création peu après de la Datar relève du même mouvement). Toutefois, il faut remonter beaucoup plus haut dans le temps pour repérer au Brésil les premières interventions de l'État fédéral spécifiques en faveur du Nordeste. C'est dès la fin du XIX siècle, suite à la sécheresse catastrophique des années 1877-79, que l'État, à l'époque l'Empire, a apporté des contributions destinées à lutter contre les effets de l'accident climatique, aides d'urgence aux populations et financement d'infrastructures hydrauliques supposées régler le problème à long terme. Pendant près d'un siècle, se déroule donc une première et longue étape de l'action étatique dans le Nordeste. Qu'il ait fallu changer d'orientation en 1960 et que cette région continue à être aujourd'hui la grande poche de pauvreté de l'Amérique latine suffiraient à dire la médiocrité des résultats, ou plutôt leur ambiguïté. Il ne s'agit pas tant en effet de lire les scores économiques et les indicateurs sociaux, ce qui n'est d'ailleurs jamais inutile, et de constater l'écart persistant entre la réalité et les objectifs visés ou les performances réalisées par les autres, mais plutôt de voir comment cette politique régionale ne pouvait avoir que des effets sociaux correctifs dans une région très inégalitaire socialement, et comment au total l'action conduite officiellement au nom de la justice a consolidé l'injustice.

Premier point, fondamental : le diagnostic du malaise régional ne portait pas sur les structures sociales, qui, selon une interprétation rawlsienne, peuvent dans le cas d'espèce être considérées comme injustes dans la mesure où elles sont contreproductives économiquement et interdisent la promotion des plus pauvres. Au lieu de quoi, les caprices du climat étant tenus pour responsables du malheur des hommes, on naturalise la question sociale et on conduit des travaux hydrauliques en laissant intacte la structure foncière. Comment alors ne pas conforter ceux qui ont la terre? Et, les travaux étant réalisés au moment des sécheresses par l'emploi des sinistrés sur des chantiers financés par l'État, comment ne pas voir que l'action dite régionale, si elle soulage effectivement en fournissant des moyens temporaires de survie, utilise la détresse des plus pauvres pour favoriser les plus riches! Parler ici de formation sociospatiale plutôt que de région aide à comprendre que l'action sur un espace est aussi une action sur les hommes, c'est-à-dire sur une société traversée d'intérêts contradictoires, de telle sorte que toute action technique engagée sans que soit au préalable retenu un projet de société alternatif a comme conséquence nécessaire de renforcer les forts aux dépens des faibles. La conduite de cette politique hydraulique impliquait la délimitation du périmètre d'intervention connu sous le nom de polygone des sécheresses. Comme toujours en pareil cas, définir la zone éligible aux aides publiques constituait un enjeu important. Restreint dans sa première version aux espaces les plus vulnérables (le Ceara, le sertao du Rio Grande do Norte, de la Paraiba et du Pernambouc), le polygone a été par la suite étiré vers le Sud, moins sensible aux accidents climatiques, mais où les latifondistes étaient eux aussi soucieux de profiter de la manne publique... 

l'industrie de la sécheresse, nom par lequel on désigne traditionnellement dans le Nordeste le profit que tirent les propriétaires fonciers des aides apportées par l'État à l'occasion des accidents climatiques, à l'industrie tout court. Cette étape aurait pu servir la justice si les orientations qu'avait fixées initialement Celso Furtado n'avaient pas été brutalement amputées de leur dimension sociale par le régime militaire issu du coup d'État de 1964: les avantages fiscaux accordés aux investissements étaient maintenus, mais était abandonnée toute perspective de transformation sociale qui, en distribuant plus équitablement la terre et le revenu, aurait permis une répartition plus juste des effets d'entraînement et donc une meilleure diffusion spatiale de la croissance. À la concentration sociale du revenu a correspondu la concentration spatiale des nouvelles activités, comme si la politique suivie visait l'insertion sélective au système productif national de seuls quelques lieux nordestins, par exemple Salvador et son pôle pétrochimique de Camaçari. Seuls les centres régionaux situés sur le littoral étaient destinés à recevoir des investissements et à devenir des périphéries intégrées, alors que l'intérieur était selon les cas, et en gardant la terminologie d'Alain Reynaud, une périphérie délaissée ou assistée... Au total, le système exclut plus qu'il n'intègre. Peuton alors parler de développement régional quand la politique socio-économique qui se trouve à l'origine des transformations spatiales n'est plus elle-même une politique de développement au sens plein du mot? On a parlé de modernisation conservatrice pour décrire l'évolution enregistrée par le Brésil tout entier à cette époque, modernisation certes, puisque la croissance est au rendez-vous, au moins pendant le miracle, et que l'investissement transforme l'appareil productif, mais modernisation conservatrice puisqu'au lieu de produire une plus grande justice sociale, elle fait de l'inégalité le moteur du succès économique. S'agissant du Nordeste, pour bien comprendre que les choses bougent, mais sans que les pauvres, c'est-à-dire la grande majorité, y trouvent leur part, on avancera ici le terme de modernisation de l'injustice.

L'instrumentalisation de l'Ouest

ne autre donnée importante de la géographie du Brésil doit ici être considérée dans la démarche qui consiste à penser la justice sociale, ou l'injustice sociale, inscrite dans un territoire et consolidée par l'organisation de ce territoire: les réserves foncières de l'Ouest.

L'opposition est classique entre une bande littorale, plus ou moins profonde, où se concentrent les hommes et les activités économiques, et le sertao, cet intérieur par définition peu peuplé qui a alimenté depuis la naissance du pays l'imaginaire collectif. Des bandeiras d'autrefois à la création de Brasilia, la même idée a été à l'œuvre d'occuper l'intérieur et de s'émanciper de cette organisation du territoire caractérisée par le gradient est-ouest de l'humanisation du milieu, par où se lit encore à la fin du XXe siècle, et sans doute encore pour longtemps, l'héritage colonial. Mais le peuplement et la mise en valeur des terres de l'Ouest n'ont nullement servi à y faire naître une société plus égalitaire. En réalité, c'est la société inégalitaire du Brésil peuplé qui s'est projetée dans le Brésil vide. L'urbanisme de Brasilia en est peut-être l'illustration la plus parlante. Si le dessin du plan-pilote évoque pour certains la liberté d'un oiseau en vol et si la majesté des perspectives exprime la hardiesse d'une nation dans sa marche à l'occupation de son territoire, la ségrégation sociale traduit physiquement le rapport qu'entretiennent avec le pouvoir les classes dominantes 
logées près des ministères et de la Place des Trois Pouvoirs, et les classes dominées, reléguées fort loin dans les villes-satellites.

La ville de Brasilia est ainsi à l'image du pays dont elle est la tête. Cette interprétation invite à poursuivre la réflexion à l'échelle de tout l'Ouest. On lancera alors l'idée que l'immensité de l'Ouest fut paradoxalement pour le Brésil à la fois une grande chance et un grand malheur. Que ce soit une chance ne requiert pas une longue démonstration: des réserves d'espace, ce sont des sols à exploiter un jour, ce sont des mines, ce sont des ressources énergétiques, c'est aussi, comme l'ont fait les États-Unis avec leur frontière, de quoi forger l'identité collective autour du fait pionnier et des valeurs fortes qui lui sont attachées : la jeunesse, l'esprit d'initiative, la volonté. Mais, au Brésil, ce fut encore le moyen de reporter toujours à plus tard les réformes qui auraient été indispensables sans l'exutoire à l'injustice sociale qu'ont pu être les réserves foncières du plateau central et de l'Amazonie. Le thème récurrent de la réforme agraire est ici éloquent. Combien de fois a-t-on entendu des responsables dire que la véritable réforme agraire dans un pays comme le Brésil, c'est la conquête des terres vierges? En d'autres termes, pourquoi partager les latifondios alors que des surfaces immenses sont libres, ou supposées telles, et attendent leur mise en valeur? D'où cette instrumentalisation de l'Ouest dans le discours politique comme solution aux tensions sociales et cet abus de langage qui fait nommer réforme agraire une opération conçue pour éviter d'avoir à faire le partage des terres.

L'Ouest peut ainsi être interprété comme la donnée naturelle qui a été utilisée pour faire perdurer l'injustice sociale. Permettant que les structures injustes se perpétuent à l'est du pays et, par voie de conséquence, empêchant qu'une dynamique sociale fondée sur une classe moyenne ne s'y mette en route et y produise un développement économique dont le ressort eût été la justice sociale, il a rendu possible une fuite en avant qui a projeté sur les terres nouvelles le malaise social et la violence. Sans même parler ici des menaces qui pèsent sur les Indiens car ce serait un autre sujet, les drames qui se vivent aujourd'hui sur la bordure amazonienne, où la lutte pour la terre met face à face les occupants sans titre et les grands propriétaires dans un combat inégal que l'État ne parvient pas à arbitrer, sont l'illustration la plus actuelle de la diffusion géographique de l'injustice.

Reste enfin que la marche pionnière perpétue le comportement dévastateur des planteurs d'autrefois. Depuis que le Brésil existe, l'Ouest a créé l'illusion d'une nature généreuse à l'infini et a fait considérer comme inutiles et anti-économiques les pratiques de conservation des sols. Exemple parmi d'autres, il en fut ainsi au XIX ${ }^{e}$ siècle à São Paulo où la dégradation des surfaces plantées en café a poussé à avancer sur les terres nouvelles, alors forestières, pour y ouvrir de nouvelles plantations. La dynamique pionnière est donc indissociable du peu de soin accordé aux équilibres écologiques. Aujourd'hui, le problème a pris une importance nouvelle avec la mise en valeur des terres amazoniennes : c'est devenu un problème de résonance mondiale posé de plus en plus en termes de développement durable, c'est-à-dire finalement en termes de justice. Dans l'idée même de développement, on l'a dit plus haut, est présente l'idée d'une certaine justice dans l'accès aux biens, et dans l'idée de développement durable est présente l'idée de la justice intergénérationnelle. La menace qui pèse sur l'environnement doit alors être comprise comme une injustice faite aux générations futures, dans le sens que le défrichement aveugle de ressources naturelles non encore répertoriées risque de priver nos descendants de biens qui pourraient leur être utiles. 
C'est donc une nécessité éthique, pour aujourd'hui et pour demain, de repenser le développement dans sa dimension environnementale, et il faut noter avec satisfaction que les autorités brésiliennes sont aujourd'hui plus sensibles à ce problème que par le passé. Est-ce parce que les dommages écologiques atteignent une gravité inédite ? Estce sous la pression internationale ? Sans doute, mais aussi et peut-être surtout parce que l'opinion brésilienne elle-même est sensibilisée à cette question. Après les années de la dictature militaire où beaucoup s'étaient laissés tromper par le mirage du Brasil grande, fait retour un comportement davantage citoyen conforme au rétablissement de la démocratie et, pour cette raison, attentif aux conséquences pour demain des choix faits aujourd'hui.

41 Cette dernière considération suggère, en matière de conclusion, de ne pas limiter la réflexion sur la justice aux seuls biens matériels.

Le respect des droits de chacun, la liberté individuelle et, donc, la possibilité offerte à tous du respect de soi-même, font partie de ce que John Rawls appelle les biens sociaux premiers, au même titre que le partage des richesses, en quoi il rejoint le principe kantien de finalité de la personne. Un autre aspect de la justice tient donc dans la participation au pouvoir et la reconnaissance de tous dans la Cité. Cela porte un nom : démocratie. Est-ce un domaine où la géographie peut parler? D'aucuns en douteront: ils laisseront la chose aux citoyens pour ce qui est de la pratique et aux politologues pour ce qui est de l'analyse. Si ce qui précède a un sens, on avancera pourtant que la géographie, en tant que science de la différenciation spatiale, est dans son rôle lorqu'elle interroge la nature de l'État et cherche à mesurer le pouvoir qu'ont les citoyens d'influer sur ses choix qui impliquent le territoire. Dire, comme ce fut fait ici, que l'État conduit une politique d'aménagement conforme aux intérêts de certains sous couvert de l'intérêt général, c'est analyser par l'entrée géographique le caractère antidémocratique et antisocial des mécanismes mis en œuvre. Constater, comme il est possible heureusement de le faire aujourd'hui au Brésil, que la démocratie progresse, c'est espérer qu'apparaisse une nouvelle façon collective d'être au territoire.

Mais la réalité n'est jamais simple ! Au moment même où la démocratie est de retour au Brésil et dans d'autres pays latino-américains, une tendance lourde au libéralisme s'impose à l'échelle mondiale. Retour de balancier après l'échec du tout-État dans les systèmes centralisés dits socialistes, cette tendance au moins d'État comporte le risque de moins de citoyenneté et de moins de démocratie, c'est-à-dire de moins de justice. D'aucuns prétendent que les deux évolutions se complètent, qu'elles sont les deux versants d'un même processus, et qu'il ne peut y avoir une démocratisation politique sans une libéralisation économique et donc une réduction de l'État. C'est là une conception de la démocratie que l'on peut ne pas partager. On peut plaider pour un État qui soit fort, efficace, ni totalitaire ni confisqué par une oligarchie, mais représentatif de la volonté générale et par conséquent légitime dans ses choix territoriaux. Faire que le territoire soit l'affaire de tous, c'est aussi cela la démocratie et la justice. 


\section{NOTES}

1.Alain Reynaud, Société, Espace et Justice, Paris, PUF, 1981.

2.Pierre Monbeig, Pionniers et planteurs de São Paulo, Paris, A. Colin, 1952.

3.John Rawls, Théorie de la Justice, Paris, Le Seuil, 1987, 666 p., trad. de l'anglais par Catherine Audard, p. 91.

4.John Rawls, op. cit., p. 93.

5.John Rawls, op. cit., p. 331.

6.Voir notamment Arghiri Emmanuel, L'échange inégal, Maspéro, 1969, et du même auteur : "Le prix 'rémunérateur', épilogue à l'échange inégal' », in Tiers-Monde, vol XXI, $\mathrm{n}^{\circ} 18$, janvier-mars 1980, p. 21-40.

7.L'expression est tirée d'Alain Reynaud (op. cit.).

8.John Rawls, op. cit., p. 131.

\section{AUTEUR}

\section{BERNARD BRET}

Maître de conférence, université de Paris-Val-de-Marne 